\title{
Possibility to Realize the Brain-Computer Interface from the Quantum Brain Model Based on Superluminal Particles
}

\author{
Takaaki Musha ${ }^{1}$, Toshiki Sugiyama ${ }^{2}$ \\ ${ }^{1}$ Advanced Science-Technology Research Organization, Namiki, Yokohama, Japan \\ ${ }^{2}$ EKBO Inc., Oigawa, Atsugi-shi, Japan \\ E-mail: takaaki.musha@gmail.com, sugit@ekbo.com \\ Received August 1, 2011; revised September 22, 2011; accepted December 1, 2011
}

\begin{abstract}
R. Penrose and S. Hameroff have proposed an idea that the brain can attain high efficient quantum computation by functioning of microtubular structure of neurons in the cytoskeleton of biological cells, including neurons of the brain. But Tegmark estimated the duration of coherence of a quantum state in a warm wet brain to be on the order of $10^{-13}$ seconds, which is far smaller than the one tenth of a second associated with consciousness. Contrary to his calculation, it can be shown that the microtubule in a biological brain can perform computation satisfying the time scale required for quantum computation to achieve large quantum bits calculation compared with the conventional silicon processors even at the room temperature from the assumption that tunneling photons are superluminal particles called tachyons. According to the non-local property of tachyons, it is considered that the tachyon field created inside the brain has the capability to exert an influence around the space outside the brain and it functions as a macroscopic quantum dynamical system to meditate the long-range physical correlations with the surrounding world. From standpoint of the brain model based on superluminal tunneling photons, the authors theoretically searched for the possibility to realize the brain-computer interface that allows paralyzed patient to operate computers by their thoughts and they obtained the positive result for its realization from the experiments conducted by using the prototype of a brain-computer interface system.
\end{abstract}

Keywords: Brain-Computer Interface, Evanescent Photon, Tachyon, Quantum Computation, Decoherence

\section{Introduction}

There are some researchers to attempt to explain the higher performance of human brains, including consciousness from the standpoint that the human brain functions as a quantum computer system proposed by Feynman. Hameroff and Penrose have constructed a theory, in which human consciousness is the result of quantum gravity effects in microtubles, which they dubbed Orch-OR (orchestrated object reduction) that involved a specific form of quantum computation conducted at the level of synapses among brain neurons [1]. Hameroff suggested that microtubules in the brain were acting as waveguides for photons and as holographic processors [2]. The cytoskeleton of biological cells, including neurons of the brain, is made up of microtubules, which are comprised of subunits of the protein, named tubulin [3].
Each tublin molecule can function as a switch between two conformations which exist in quantum superposition of both conformational states.

According to Jibu et al. [4,5], microtubule quantum states link to those of other neurons by quantum coherent photons tunneling through membranes in biological systems and the cytoskeletal protein conformational states are entangled by these photons which form coherent domains in their interaction with the local electromagnetic field. According to their hypothesis of quantum brain, microtubule quantum states link to those of other neurons by quantum coherent photons tunneling through membranes in biological systems functioning in a way resembled as an ion trap computers. However, Max Tegmark [6] published a refutation of the Orch-OR model in his paper that the time scale of neuron firing and excitations in microtubules was slower than the de- 
coherence time by at least $1 / 10^{10}$. According to his paper, it is reasonably unlikely that the brain functions as a quantum computer at room temperature. Contrary to the Tegmark's calculation, Musha [7] has shown that the microtubule in a biological brain can perform computation satisfying the time scale required for quantum computation to achieve large quantum bits computation compared with the conventional silicon processors even at the room temperature from the assumption that the evanescent photon is a superluminal particle called tachyon.

A brain-computer interface (BCI), sometimes called a direct neural interface or brain-machine interface, is a direct communication pathway between a brain and an external device. Based upon the brain model by superluminal evanescent photons, the authors searched for the possibility to realize the brain-computer interface from the theoretical analysis and they also conducted the experiment to confirm the theory by using the manufactured BCI system.

\section{Theoretical Possibility of the Brain-Computer Interface System}

\subsection{Possibility of Quantum Superposition outside the Biological Human Brain}

M. Jibu et al. [8] proposed that the conscious process in the brain was related with the macroscopic condensates of massive evanescent photons generated by the Higgs mechanism, i.e. that of general biological cell functioning arising from dynamical effects of electromagnetic interaction among electric dipoles in biological systems. They claimed that human consciousness could be understood as arising from those creation-annihilation dynamics of a finite number of evanescent (tunneling) photons in the brain. E. Recami [9] claimed in his paper that tunneling photons traveling in an evanescent mode can move with superluminal group speed, which can be shown as follows.

The evanescent photon generated in quantum domain satisfies the following Klein-Fock-Gordon equation given by

$$
\left[-\frac{1}{c^{2}} \frac{\partial^{2}}{\partial t^{2}}+\nabla^{2}-\frac{m_{0}^{2} c^{2}}{\hbar^{2}}\right] \varphi(x, t)=0,
$$

where $c$ is the light speed, $m_{0}$ is an absolute value of the proper mass of the evanescent photon and $\hbar$ is the Planck constant divided by $2 \pi$.

This equation has the solution for the photon traveling in an evanescent mode given by

$$
\varphi(x, t)=A_{0} \exp \left[-\frac{E t+p x}{\hbar}\right],
$$

which corresponds to the elementary particle with an imaginary mass $i m_{0}$ that travels at a superluminal speed satisfying

$$
E^{2}=p^{2} c^{2}-m_{0}^{2} c^{4}
$$

where $E$ is the energy of the superluminal particle and $p$ is its momentum given respectively as

$$
E=\frac{m_{0} c^{2}}{\sqrt{v^{2} / c^{2}-1}},
$$

and

$$
p=\frac{m_{0} v}{\sqrt{v^{2} / c^{2}-1}} .
$$

Hence, it can be seen that tunneling photons traveling in an evanescent mode can move at a superluminal speed. Therefore it seems highly plausible that it is not the microscopic quantum mechanical system of electrons but the macroscopic quantum ordered dynamical system of evanescent photons in the brain plays the essential role in realizing long-range biological order in living systems. Ziolkowshi pointed out in his paper that superluminal pulse propagation which permits consequent superluminal exchange without a violation in causality is possible in the electromagnetic metamaterial [10]. If inner medium of the cylinder of microtubule possesses the characteristics of metamaterial with negative refractive index, the tunneling photons are actually enhanced and propagates losslessly inside the neurons not restricted by its wavelength, and the infrared photon can be used for the manipulation of qubits in the brain.

Laszlo [11] pointed out the puzzle in his book that the living organism is extraordinary coherent with the world around it, dynamically, almost instantly correlated with all other parts, shown as follows;

- The mind of one person appears able to act on the brain and body of another.

- Modern people display a capacity for spontaneous transference of impression and images, especially when they are emotionally close to each other.

This is impossible if the human brain functions similar to the conventional computer systems using silicon processors. The level of coherence exhibited by organism suggests that quantum type processing take place in them. The living organism, it appears, is in some respects a macroscopic quantum system.

If the brain is functioned by the superluminal particles called a tachyon, this puzzle can be clarified.

From the Klein-Fock-Gordon equation for a scalar field with an imaginary mass $m=i m_{0}$ given by

$$
\left(\frac{\partial^{2}}{\partial t^{2}}-\nabla^{2}-m^{2}\right) \phi=0,
$$


where $m=m_{0} c / \hbar$. From which, we can obtain an elementary solution shown as

$$
\phi_{k}(x)=\frac{1}{(2 \pi)^{3 / 2}} \exp [i(k \cdot x-\omega t)] \equiv \frac{1}{(2 \pi)^{3 / 2}} \exp (i k x),
$$

where $\omega=\sqrt{k^{2}-m_{0}^{2}}$. Thus it can be seen that tachyons cannot be localized in space from the superposition of solutions given by Feinberg shown as [12].

$$
\phi(x)=\int \phi_{k}(x) f(k) \mathrm{d}^{3} k \neq \delta^{3}(x),
$$

where $f(k)$ is a function of bounded support, $\varphi_{k}$ is a set of elementary solutions of the Klein-Fock-Gordon equation with an imaginary mass and $\delta(x)$ is the Dirac's delta function. Thus such a quantum superposition cannot be made to vanish outside the sphere of finite radius, but rather has a finite tail which is made to decrease with an arbitrary power of $x$ for large values, by choosing the weight function $f(k)$ to have a zero of suitable order at $|k|=m_{0}$. If the brain is a quantum computer system functioned by superluminal photons, it can be entangled with each other via the tachyon field created from the quantum vacuum around it according to the property of non-locality to exert influences outside the extent of the biological brain as shown in Figure 1.

\subsection{Quantum Coherence between the Tachyon Field and Electrons in a PC Processor}

A particle interaction with a scalar field through the Hamiltonian has been studied for the case, in which only thermal-excitation effects of the scalar field are taken into account and the effect of zero-point vacuum fluctuations is neglected [13].

According to Zurek, the density matrix $\rho\left(x, x^{\prime}\right)$ of the particle in the position representation evolves by the master equation shown as [14]

$$
\begin{aligned}
\dot{\rho}= & -\frac{i}{\hbar}[H, \rho]-\gamma\left(x-x^{\prime}\right)\left(\frac{\partial}{\partial x}-\frac{\partial}{\partial x^{\prime}}\right) \rho, \\
& -\frac{2 m \gamma k_{B} T}{\hbar^{2}}\left(x-x^{\prime}\right)^{2} \rho
\end{aligned}
$$

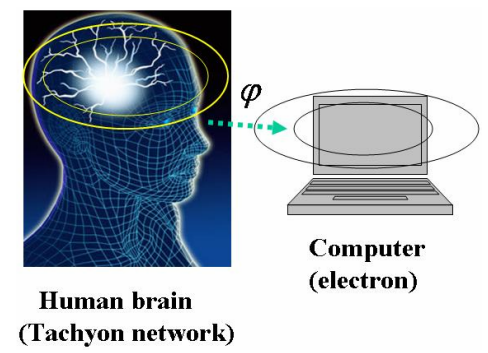

Figure 1. Tachyonic field in the brain which affect wave functions of electrons in a computer system. where $H$ is the particle's Hamiltonian, $\gamma$ is the relaxation rate, $k_{B}$ is the Boltzmann constant, and $T$ is the temperature of the field.

Zurek has shown that the off-diagonal peaks of the density matrix, $\rho\left(x, x^{\prime}\right)=\varphi(x) \varphi^{*}\left(x^{\prime}\right)$ will decay at the rate

$$
\frac{\mathrm{d}}{\mathrm{d} t}\left(\rho^{ \pm}\right) \approx 2 \gamma m k_{B} T / \hbar^{2}(\Delta x)^{2} \rho^{ \pm}=\rho^{+} / \tau_{D} .
$$

From which, quantum coherence will disappear on a time scale shown as

$$
\tau_{D} \cong \tau_{R}\left(\frac{\hbar}{\Delta x \sqrt{2 m k_{B} T}}\right)^{2}
$$

The decoherence time for the quantum computation utilizing superluminal particle, Musha has shown the formula in his paper shown as [7]

$$
\tau_{D}^{\prime} / \tau_{D} \approx[\beta(\beta-1)]^{2},
$$

where $\tau_{D}^{\prime}$ is the decoherence time for the superluminal particle. In this formula $\beta$ is the rate of the velocity of the superluminal particle relative to the light speed shown as

$$
\beta \approx 1+\frac{c}{2 \omega d}+\sqrt{\frac{c}{\omega d}+\frac{c^{2}}{4 \omega^{2} d^{2}}},
$$

where $\omega$ is an angular frequency of the particle and $d$ is the size of the tunneling barrier. When we suppose that the tachyon field can be generated in a microtubule in a human brain with the frequency at the infra-red spectrum, which wavelength is given by $\lambda=100 \mu \mathrm{m}$, we have $\tau_{D}^{\prime} / \tau_{D}=1.3 \times 10^{12}$ for the case when we let $d=15 \mathrm{~nm}$; that is the same order as the extracellular space between the brain cells.

Then the decoherence time for the tachyon field in a brain which influences wave function of electrons in a $\mathrm{PC}$ processor is given by

$$
\tau_{D}^{\prime} \approx \tau_{R}\left(\frac{\hbar}{\Delta x \sqrt{2 m k_{B} T}}\right)^{2}[\beta(\beta-1)]^{2} \approx 2 \times 10^{-4} \tau_{R}(\mathrm{sec}),
$$

When we set $m=9.11 \times 10^{-31} \mathrm{Kg}, T=300^{\circ} \mathrm{K}$ and $\Delta x=10 \mathrm{~cm}$. If the relaxation time were of the order of macroscopic time satisfying $\tau_{R} \gg 1 \mathrm{sec}$, quantum coherence would be destroyed in $\tau_{D}^{\prime} \gg 2 \times 10^{-4} \mathrm{sec}$, which might be sufficient to affect wave functions of electrons in the PC processor. In a semiconductor, the mass of electrons can be replaced by the effective mass given by $0.258 \mathrm{~m}$, the decoherence time can be postponed about 4 times longer than the free electron case accord- 
ing to Equation (14) and it is strongly supported that human consciousness can influence the PC processor via the tachyon field generated inside the brain.

\section{Experimental Results by Using the Trial Manufactured BCI System}

According to an idea that human consciousness can influence electrons in the PC processor via the tachyon field generated inside the brain, the Brain Computer Interface (BCI) system composed of the $\mathrm{PC}$ processor that can generate serial data at 2500 bit per second, was manufactured by one of the authors, Sugiyama, as shown in Figure 2, the block diagram of which is shown in Figure 3.

As shown in the block diagram, the noise source generates pulses with the randomized length of pulse and they are supplied to the gate circuit and then sent to the pulse counter, which counts the pulse number within a finite length of time. It is designed to output the low level of voltage for the case when the count number is even and output the high level for the case when the count number is odd at the terminal of this electric circuit.

From which, the curve of total sum can be constructed on the computer display. The curve is composed of 20000 bit data as a total sum and the time duration of which is 8 seconds. If there in no disturbance from outside, we can observe the curve of random walk on the display according to the probability theory. Supposing that the human consciousness can influence the movement of electrons in the computer processor, the accumulation curve will shows a different characteristics from the random walk as predicted by the theory of random walk that standard deviation scales increase in proportional to the square root of bit number of the data as shown in Figure 4. At the experiment, the subjects kept in mind two cases such as "converge" or "spread" of the data shown on the display. Figure 5 shows some examples of the experimental result when subjects kept in their mind to converge the data.
Table 1 shows the statistical values obtained by six trials. From which, we can recognize the influence of the human consciousness on the total curve on the PC by the consciousness of the person under trials.

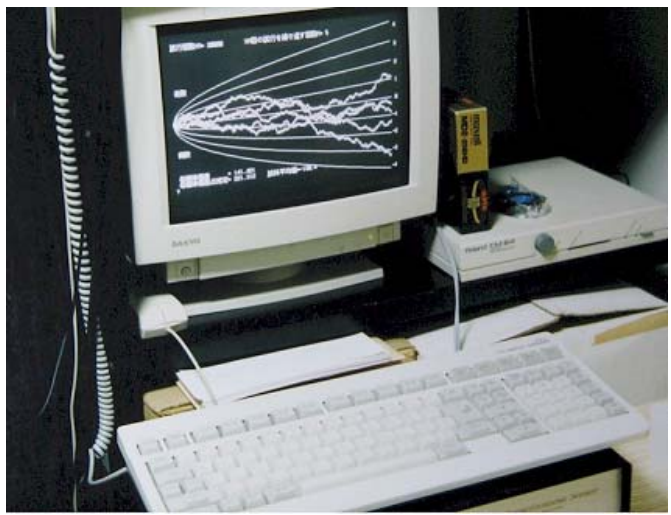

Figure 2. Photo of the manufactured BCI system.

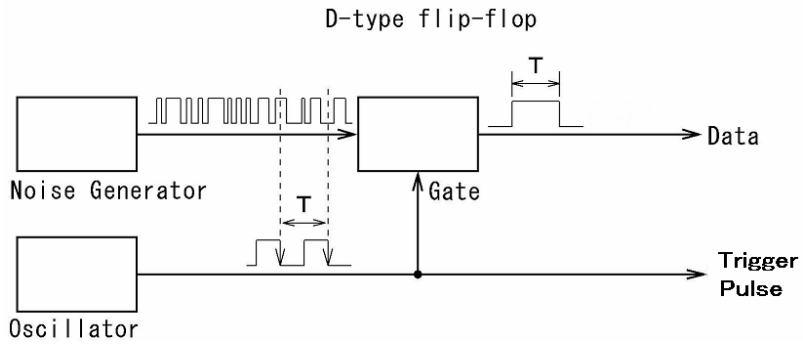

Figure 3. Block diagram of the BCI system.

Table 1. Experimental results.

\begin{tabular}{cccccc}
\hline No & $\begin{array}{c}\text { Standard } \\
\text { deviation }\end{array}$ & $\begin{array}{c}\text { Sample } \\
\text { Variance (A) }\end{array}$ & $\begin{array}{c}\text { Unbiased } \\
\text { variance (B) }\end{array}$ & B/A & Command \\
\hline 1 & 141.4 & 20000 & 8325.2 & 0.42 & converge \\
2 & 141.4 & 20000 & 2436.0. & 0.12 & converge \\
3 & 141.4 & 20000 & 5513.2 & 0.28 & converge \\
4 & 141.4 & 20000 & 3891.2 & 0.19 & converge \\
5 & 141.4 & 20000 & 31657.3 & 1.58 & spread \\
6 & 141.4 & 20000 & 39621.3 & 1.98 & spread \\
\hline
\end{tabular}

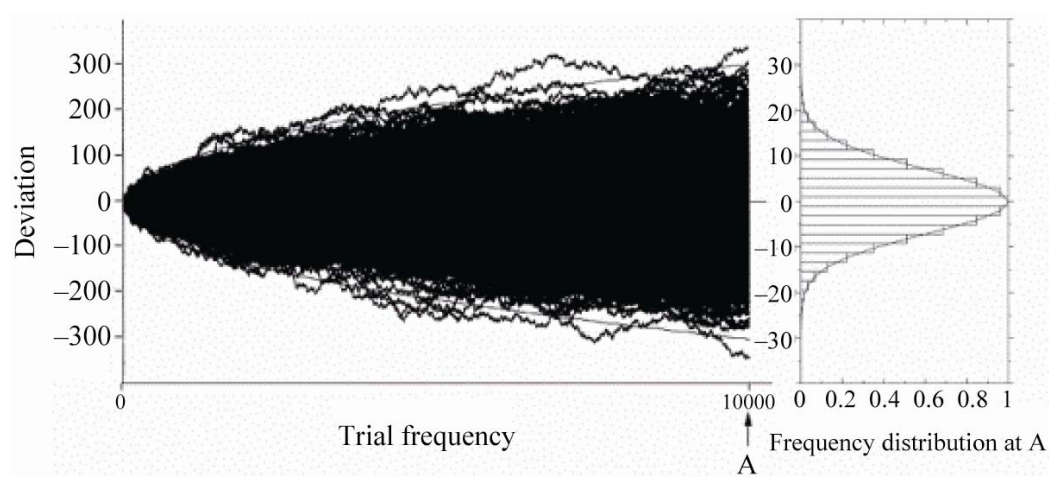

Figure 4. Display of the BCI system according to the random walk theory. 


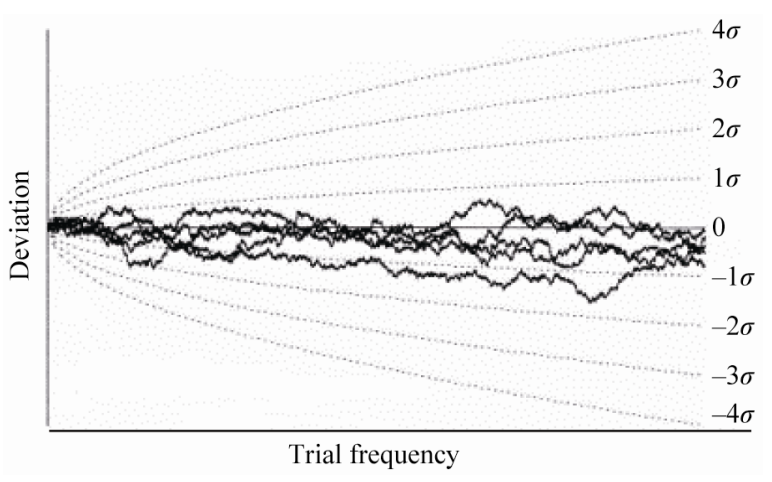

Figure 5. Experimental result by the BCI system.

From these experimental results, it is considered that the human consciousness can influence the computer function as predicted by the brain model based on the superluminal particles. Thus it is considered that these results open the door to the possibility to achieve the brain-computer interface to operate computers of move robotic arms using nothing but human's thoughts.

Further experiments conducted by using the random data sampled about an hour ago to display on the screen showed a similar result that the accumulation curve was changed according to the human will on real time. This result showed that the human will affected the computer function beyond time. This result has shown the violation of causality, which confirms our hypotheses that consciousness is a field of tachyonic or superluminal matter located beyond the barrier of the light. Some researchers such as Prof. R. Dutheil proposed the same idea that our consciousness or the mind is composed of tachyons located in superluminal space-time and our subliminal universe is merely a holographic projection of the fundamental universe, where information and meaning are located [15]. From the application of superluminal human consciousness, it is considered that there is a possibility to develop a computer-brain interface with higher capabilities than the conventional electric systems.

\section{Conclusions}

Based upon the brain model that consciousness is composed of superluminal evanescent photons, the authors searched for the possibility to realize the brain-computer interface both by the theoretical analysis and the experiment, and they obtained the positive result for its realization. Hence it is considered that that there is a possibility to develop a computer-brain interface which can manipulate electric systems by thought alone. By realizing the interface between the brain and the computer, it will help persons who are unable to move or speak to resume their interaction with the world around them.

\section{References}

[1] S. Hameroff and R. Penrose, "Conscious Events as Orchestrared Space-Time Selections," Journal of Consciousness Studies, Vol. 3, No. 1, 1996, pp. 36-53.

[2] S. Hameroff, "Quantum Computation in Brain Microtubules? The Penrose-Hameroff 'Orch OR' Model of Consciousness," Philosophical Transactions Royal Society London (A), Vol. 356, No. 1743, 1998, pp. 18691896.

[3] S. Hameroff, "Information Processing in Microtubules," Journal of Theoretical Biology, Vol. 98, No. 4, 1992, pp. 549-561. doi:10.1016/0022-5193(82)90137-0

[4] M. Jibu and K. Yasue, "What Is Mind? Quantum Field Theory of Evanescent Photons in Brain as Quantum Theory of Consciousness," Informatica, Vol. 21, No. 3, 1997, pp. 471-490.

[5] M. Jibu, K. Yasue and S. Hagan, "Evanescent (Tunneling) Photon and Cellular Vision," BioSystems, Vol. 42, No. 1, 1997, pp. 65-73. doi:10.1016/S0303-2647(97)01686-9

[6] M. Tegmark, "Importance of Quantum Coherence in Brain Processes," Physical Review E, Vol. 61, No. 4, 2000, pp. 4194-4206. doi:10.1103/PhysRevE.61.4194

[7] T. Musha, "Possibility of High Performance Quantum Computation by Superluminal Evanescent Photons in Living Systems," BioSystems, Vol. 96, No. 3, 2009, pp. 242-245. doi:10.1016/j.biosystems.2009.03.002

[8] M. Jibu, K. H. Pribram and K. Yasue, "From Conscious Experience to Memory Storage and Ritrieval: The Role of Quantum Brain Dynamics and Boson Condensation of Evanescent Photons," International Journal of Modern Physics B, Vol. 10, No. 13\&14, 1996, pp. 1753-1754. doi:10.1142/S0217979296000805

[9] E. Recami, "Superluminal Motions? A Bird-Eye View of the Experimental Situation," Foundations of Physics, Vol. 31, No. 7, 2001, pp. 1119-1135. doi:10.1023/A:1017582525039

[10] R. W. Ziolkowski, "Superluminal Transmission of Information through an Electromagnetic Metamaterial," Physical Review E, Vol. 63, No. 4, 2001, pp. 046604-046617. doi:10.1103/PhysRevE.63.046604

[11] E. Lazlo, "Science and the Akashic Field: An Integral Theory of Everything," Inner Traditions, Rochester, 2004.

[12] G. Feinberg, "Possibility of Faster-Than-Light Particles," Physical Review, Vol. 159, No. 5, 1967, pp.1089-1100.

[13] B. Duplantier, J.-M. Raimond and V. Rivasseau, "Quantum Decoherence, Poincare Seminar 2005," Birkhäuser Verlag Basel, Berlin, 2006.

[14] W. H. Zurek, "Decoherence and the Transition from Quantum to Classical-Revisited," Los Alamos Science, No. 27, 2002, pp. 2-25.

[15] E. E. Valarino, "On the Other Side of Life, Exploring the Phenomenon of the Near-Death Experience," Perseus Publishing, Cambridge, 1997. 